\title{
Acne excoriée als Manifestation einer Dermatitis artefacta
}

\author{
A.T. Köhler ${ }^{1}$ \\ C. Alliger-Horn ${ }^{2}$ \\ R. Hartmann ${ }^{1}$ \\ H. Neuber ${ }^{1}$
}

\section{Excoriated Acne: A Manifestation of Dermatitis artefacta}

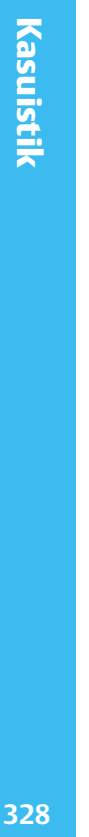

\section{Zusammenfassung}

Kasuistik eines 36-jährigen Mannes mit jahrelang bestehenden Hautveränderungen im Gesicht. Aufgrund der Anamnese, Normalbefund aller Untersuchungsergebnisse, typischer Kriterien und rascher Abheilung unter abdeckenden Wundauflagen konnte die Diagnose Dermatitis artefacta gestellt werden. In der Diskussion werden das Spektrum artefizieller Hautläsionen und Kriterien der Diagnostik der Dermatitis artefacta vorgestellt. Häufig leiden die Betroffenen unter einer Borderline-Störung, die therapeutisch schwer zugänglich ist. Im Rahmen interdisziplinären Vorgehens nahm bei dem Patienten die eingeleitete Psychotherapie einen günstigen Verlauf.

\section{Abstract}

Case report of a 36 year old male with chronic facial skin lesions. History, normal investigative findings, typical criterias and rapid healing under occlusive dressings led to the diagnosis Dermatitis artefacta. Criterias for factitious syndromes and clinical picture of Dermatitis artefacta are presented. A high number of patients suffers from borderline personality disorder. In the vast majority of patients the condition is chronic. In this case interdisciplinary efforts led to successful psychiatric referral.

\section{Einleitung}

Gewöhnlich handelt es sich bei artefiziellen Störungen um eine Ausschlussdiagnose, die erst nach gewissenhafter organmedizinischer Abklärung gestellt werden darf. Über ihre Häufigkeit im Krankengut gibt es nur Schätzungen [7]. Die Diagnose zu stellen ist schwierig, ein Rest Unsicherheit bleibt vielleicht bestehen, und aufgrund der manchmal mühevollen Arzt-Patient-Beziehung beschränkt man sich allzu gerne auf die Linderung rein somatoformer Störungen. Die nachfolgende Kasuistik aus dem eigenen Patientengut soll dieses Krankheitsbild näher erläutern.

\section{Kasuistik}

\section{Anamnese}

Der 36-jährige Mann stellt sich seit über einem Jahr regelmäßig in der Poliklinik vor. Seine chronischen Hautveränderungen begannen 1997 und bestehen seither fort, dabei liegt ein wechselhafter, überwiegend therapierefraktärer Verlauf vor. Seit 1997 besucht er regelmäßig die Ambulanzen zweier dermatologischer Polikliniken und wurde einmal konsiliarisch in der Ambulanz einer Universitäts-Hautklinik vorgestellt (Abb.1).

Je nach Aspekt und Ausdehnung des Hautbefunds ist dabei von oberflächlichen Erosionen, Pyodermie unklarer Genese, Acne excoriée oder diskoidem Lupus erythematodes die Rede. Zahlreiche Erregerabstriche und aufwändige bakteriologische Untersuchungen stehen einer nur einmal durchgeführten Probeentnahme gegenüber. Unterschiedliche systemische und topische The-

Institutsangaben

${ }^{1}$ Abteilung Dermatologie, Bundeswehrkrankenhaus Berlin (Leiter: Dr. A. Zimmerlinkat)

${ }^{2}$ Abteilung Neurologie und Psychiatrie, Bundeswehrkrankenhaus Berlin (Leiter: Dr. H.-H. Hahne)

Korrespondenzadresse

Dr. med. Axel T. Köhler · Abteilung Dermatologie · Bundeswehrkrankenhaus Berlin ·

Scharnhorststraße $13 \cdot 10115$ Berlin 
rapien, u.a. Antibiotika, Antiseptika, Isotretinoin und Dapson, führen langfristig zu keiner Abheilung, obwohl der Patient die therapeutischen Maßnahmen bereitwillig und scheinbar zuverlässig durchführt. Der Patient arbeitet seit über einem Jahr als Assistent in einem pharmazeutischen Labor. Den Ursachen seiner Erkrankung steht er ratlos gegenüber, gelegentlich beschuldigt er die angebliche Exposition von „giftigen Dämpfen“ im Labor als Auslöser.

\section{Hautbefund}

Zentrofazial, auf Stirn und Glabella, am Nasenrücken und Nasenabhang imponieren flächenhafte nicht juckende Erosionen, die zum jeweiligen Randbereich hin oberflächlicher werden. Abgeheilte Areale, die teilweise leicht vernarben, wechseln ständig mit neuen Läsionen. Das übrige Integument ist erscheinungsfrei. Dermographismus ruber (Abb. 2).

\section{Histologie vom Nasenrücken Oktober ${ }^{1996}{ }^{1}$}

Unauffällig konfigurierte Epidermis, leichte follikuläre Hyperkeratose, im oberen Corium leichte Fibroplasie, einige ektatisch erweiterte Gefäße sowie angeschnittene Talgdrüsenhyperplasie. Am ehesten fibröse Nasenpapel.

\section{Bakteriologische Diagnostik}

Überwiegend Micrococcus spp. und E. coli.

\section{Therapie und Verlauf}

Der überdimensionale Umfang seiner Krankenakte und die ausführliche Dokumentation stehen im Widerspruch zur scheinbaren Harmlosigkeit der vermuteten Erkrankung. Der Wechsel der inzwischen zahlreich verdächtigen Diagnosen findet gewöhnlich dann statt, wenn sich die aktuelle Therapie in ihrem Verlauf als wirkungslos erweist.

Im Rahmen einer im Jahre 2000 vom Patienten gewünschten Laserkorrektur abgeheilter und oberflächlich vernarbter Areale kommt es durch den postoperativ aufgebrachten Okklusivverband zur raschen Abheilung der Erosionen. Die restlichen Herde heilen unter hydrokolloidalen Wundauflagen ab, daneben entstehen in nicht abgedeckten Abschnitten des Gesichts neue Läsionen.

Im Rahmen der nun folgenden ärztlichen Gespräche äußert der Patient stets den Wunsch nach umfassender organmedizinischer Abklärung seiner Hautveränderungen. Jede Form des Eigenverschuldens an der Entstehung seiner Läsionen im Gesicht lehnt er ab. Lenkt man das Gespräch von den Hautveränderungen ab auf andere Probleme in seinem Alltag, berichtet er von zahlreichen inneren Konflikten und psychosozialem Stress sowohl im Privatleben als auch am Arbeitsplatz. Völlig unerklärlich ist ihm die Tatsache, dass er gelegentlich am Morgen von seinem Gesicht mechanisch abgelöste Wundauflagen neben sich im Bett vorfindet. Lediglich unter dem Aspekt, unter einer ihn psychisch belastenden „Hautkrankheit“ zu leiden, wird er dazu gebracht, ein Gespräch mit einem Psychologen zu führen.

${ }^{1}$ Dermatohistologisches Labor Prof. H. Mensing, Heegbarg 25, 22391 Hamburg.

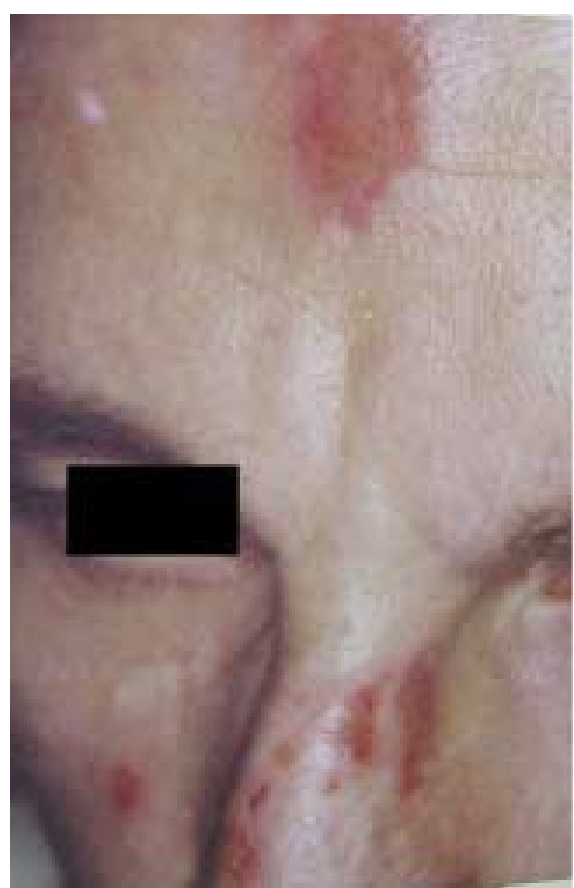

Abb. 1 Oberflächliche, zu den Randbereichen hin abflachende Erosionen an Stirn und Nasenrücken. Hautbefund 1999.

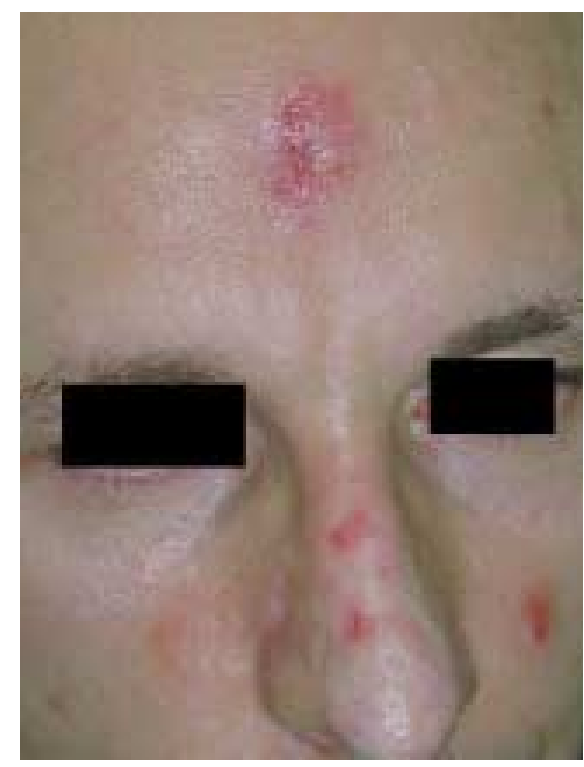

Abb. 2 Weitgehend unveränderter Hautbefund, wenige Wochen vor Beginn einer Psychotherapie im Frühjahr 2001.

\section{Psychologischer Aufnahmebefund}

Die Hautveränderungen treten vermehrt nach 1996 auf, dem Jahr der Trennung von der Lebensgefährtin und von dem gemeinsamen Kind. Frühe Interaktionsstörung in der Mutter-KindBeziehung u. a. bedingt durch die Geschwisterreihenfolge. In den psychologischen Tests zum Persönlichkeitsprofil zeigt sich bei ihm eine starke Diskrepanz zwischen verbalem und nonverbalem Ausdruckserleben und eine geringe Diskriminationsfähigkeit im emotionalen Ausdruckserleben bei sich und in der Interaktion und die Unfähigkeit, eigene Gefühle angemessen zu spiegeln (Alexithymie).

Seine Borderline-Beziehungsanteile äußern sich in Kontaktschwäche, Aggressionshemmung und tiefgreifenden dynamischen Ich-Destabilitäten. Insbesondere in (den häufigen) Phasen psychosozialer Konflikte kommt es zu einem Aufblühen der Hautveränderungen. 
Nach interdisziplinärer Beratung wird die Diagnose Dermatitis artefacta bei zugrunde liegender Borderline-Störung gestellt. Eine Psychotherapie auf der Grundlage einer Verhaltenstherapie wird eingeleitet und später durch eine analytische Gruppentherapie erweitert. Gelegentliche aber regelmäßige Konsultationen des Dermatologen auf dem Boden einer empathischen Arzt-Patient-Beziehung dienen der Beurteilung des Ausmaßes der Hautveränderungen und des Therapieerfolgs. Nach einem halben Jahr kommt es allmählich zu einem dauerhaften Rückgang der Läsionen.

\section{Diskussion}

Artifiziellen Läsionen liegen unterschiedliche psychopathologische Ursachen zugrunde (Tab.1). Bei Dermatitis artefacta fügen sich die Betroffenen bewusst oder unbewusst Selbstbeschädigungen zu, um ein psychisches Bedürfnis zu befriedigen und Zuwendung zu erfahren. Gelegentlich werden sich die Patienten ihrer selbstbeschädigenden Aktivität bewusst, häufig jedoch findet sie unkontrolliert und unbewusst statt. Gewöhnlich weisen die Betroffenen jegliche Form des Eigenverschuldens von sich. Die Diagnose fügt sich dem Arzt aus bestimmten Beobachtungen zusammen. Überdurchschnittlich häufig arbeiten die Patienten in Gesundheitsberufen. Sie haben bereits zahlreiche Spezialisten konsultiert, führen eine dicke Krankenakte angefüllt mit Normalbefunden und haben unzählige systemisch oder topisch wirksame Medikamente angewendet, die bislang allesamt nicht geholfen haben. Die sich selbst zugefügten Läsionen können in Form und Umfang sehr differenzieren, selbst einstmals bestandene Hautkrankheiten treten wieder auf oder werden nachgeahmt. Trotz grenzenloser Vielfalt in den Methoden sich zu beschädigen, ist den Patienten Ursache und Entstehung ihrer Hautveränderungen ein völliges Rätsel. Während Außenstehende oder Dritte die Läsionen und Hautveränderungen als entstellend oder schmerzbringend empfinden, zeigen sich die Betroffenen davon weniger beeindruckt $[4,5]$ (Tab. 2 ).

Die überwiegende Zahl der Betroffenen leidet an einer Borderline-Störung. Deren Ursache kann im Fehlen emotionaler Zuwendung im Säuglingsalter und in regelmäßiger Erfahrung physischen oder sexuellen Missbrauchs liegen. Die emotionale Leere und das fehlende Ausdruckserleben münden in Depression und schwachen Impulskontrolle, zwischenmenschliche Beziehungen erhalten einen manipulativen Charakter. Die inneren Spannungen der Patienten werden durch Akte der Selbstbeschädigung erleichtert. Die Krankenrolle gewährleistet Abhängigkeit und Versorgung insbesondere während stressreicher Lebenssituationen, die Hautläsionen stellen personelle und sexuelle Identität her, an Stelle von Leere und Depression treten Stimulation und Manipulation. Die verständliche Sorge des Arztes, bei dem Patienten eine organische Erkrankung zu übersehen, sowie das Drängen und die hohe Bereitschaft des Patienten, an jeglicher Diagnostik und Therapie offenbar motiviert teilzunehmen, münden in fortwährende Beanspruchung medizinischer Leistungen $[2,6,9]$.

Bei dem geschilderten Fall traten die Läsionen zeitlich gesehen nach dem Zusammenbruch seiner bisherigen sozialen Beziehungen auf. Im Sinne der Pathomimikry arbeiteten die behandelnden Ärzte im Laufe der Jahre alle möglichen Differenzialdiagnosen diagnostisch und therapeutisch ab [1].
Tab. 1 Klassifikation artefizieller Läsionen

\begin{tabular}{|c|c|}
\hline 1. Simulation & $\begin{array}{l}\text { Bewusst zugefügte Selbstbeschädigung, } \\
\text { bewussten sekundären Gewinns wegen }\end{array}$ \\
\hline 2. Hypochondrie & $\begin{array}{l}\text { Bewusst zugefügte Selbstbeschädigung, } \\
\text { die eine irrtümlich angenommene } \\
\text { Störung beheben soll }\end{array}$ \\
\hline $\begin{array}{l}\text { 3. Obsessionen, } \\
\text { Zwangsimpulse }\end{array}$ & $\begin{array}{l}\text { Ständig wiederholende Handlung mit } \\
\text { selbstbeschädigender Tendenz wie } \\
\text { Kratzen, Reiben, Haare zupfen ... }\end{array}$ \\
\hline 4. Dermatitis artefacta & $\begin{array}{l}\text { Bewusst oder unbewusst zugefügte } \\
\text { Läsionen zur Befriedigung unbewusster } \\
\text { Bedürfnisse }\end{array}$ \\
\hline $\begin{array}{l}\text { 5. Münchhausen-Syndrom } \\
\text { (i.S. artefizieller Läsionen) }\end{array}$ & $\begin{array}{l}\text { Ständig wiederholte Methoden der } \\
\text { Selbstbeschädigung, um ein unbewusstes } \\
\text { Bedürfnis zu befriedigen }\end{array}$ \\
\hline
\end{tabular}

Tab. 2 Diagnostische Kriterien Dermatitis artefacta

- Frauen > Männer
- Betroffene häufig in Gesundheitsberufen tätig
- Ungewöhnliche, in sich widersprüchliche Anamnese
- Vielzahl an diagnostischen Befunden, darunter zumeist Normalbefunde
- Völlige Wirkungslosigkeit jeglicher bisheriger Therapie
- Trotz zahlreich konsultierter Spezialisten ist die Entstehung der Läsionen
den Betroffenen völlig unklar
- Drängen auf organmedizinische Abklärung, dabei forderndes und
manipulatives Auftreten
- Diskrepanz zwischen Aspekt des Hautbefundes und subjektivem
Beschwerdebild

Auf der Suche nach einem geeigneten therapeutischen Ansatz dienen Okklusivverbände der Diagnostik aber nicht der Heilung. Externa, Umschläge, blande Salben und hautpflegende Maßnahmen können zunächst den emotional besetzten Manipulationsdruck lindern. Die unmittelbare Konfrontation und die Anschuldigung der Selbstbeschädigung führen meist dazu, dass die Liste der erfolglos konsultierten Ärzte um einen weiteren Namen verlängert wird. Eine erfolgreiche Annäherung an Betroffene gelingt nur in einem Klima der Empathie und Unterstützung. Dazu dienen mehrere, möglichst kurzdauernde Kontakte, in denen der Arzt auch etwas über die Lebensgeschichte des Patienten erfährt. Diese Gespräche lenken allmählich auf die psychosomatische Entstehung der Hautveränderungen, und manchmal gelingt es, den Patienten einer geeigneten Psychotherapie zuzuführen $[3,8]$.

Das Besondere an der geschilderten Kasuistik ist die Tatsache, dass der Patient zu einer psychotherapeutischen Intervention bereit und motiviert war. In vielen Fällen hingegen gelingt zwar keine Heilung, wohl aber eine unterstützende und empathische Führung des Patienten, die seine selbstbeschädigenden Handlungen eingrenzen hilft [4]. 
Literatur

${ }^{1}$ Azurdia RM, Guerin DM, Sharpe GR. Recurrent bullous dermatitis artefacta mimicking immunobullous disease (letter). $\mathrm{Br} J$ Dermatol 2000; 143: 229-230

2 Bosse K. Die Integration der Psychologie in die Dermatologie-Erfahrungen und Möglichkeiten. Z Hautkr 1990; 65: 419-421

${ }^{3}$ Gieler U, Effendy I, Stangier U. Kutane Artefakt-Behandlungsmöglichkeit und ihre Grenzen. Z Hautkr 1987; 62: 882 - 890

${ }^{4}$ Koblenzer CS. Neurotic excoriations and dermatitis artefacta. Psychodermatology 1996; 14: 447-455
${ }^{5}$ Koblenzer CS. Psychological aspects of skin disease. In: Fitzpatrick's Dermatology in General medicine. New York: McGraw-Hill, 1993

${ }^{6}$ Linehan MM. Cognitive-behavioral treatment of Borderline Personality Disorder. Guilford Publications, 1993

7 Oostendorp I, Rakoski J. Münchhausen-Syndrom. Hautarzt 1993; 44: $86-90$

8 Plewig G, Kligman AM. Excoriations in acne. Psychological aspects of acne. In: Plewig G (Hrsg). Acne and Rosacea. Berlin: Springer, 2000

${ }^{9}$ Senf W, Broda M. Praxis der Psychotherapie. Stuttgart: Thieme, 2000

\section{Buchbesprechung}

\section{Haut- und Vaginalmykosen.}

H.-J. Tietz, J. Mendling

143 Seiten, 68 Abb., 60 Tab. Blackwell Wissenschafts-Verlag,

Berlin 2001. Kart. DM 68,36. ISBN 3-89412-474-1

Gemessen an ihrer Häufigkeit zählen Mykosen zu den bedeutendsten Infektionskrankheiten unserer Zeit, mit der nahezu jeder Arzt in seiner täglichen Praxis konfrontiert werden kann. Obwohl die ätiologische Bedeutung der Pilze als Krankheitserreger bereits seit Mitte des 19. Jahrhunderts bekannt ist, müssen auch heute noch beachtliche Lücken im Wissen um die von Pilzen verursachten Krankheiten konstatiert werden. Einen erfolgreichen Versuch, zum Schließen dieser Wissenslücken beizutragen, unternehmen die Autoren Hans-Jürgen Tietz und Jürgen Mendling mit ihrem Buch „Haut- und Vaginalmykosen“. In kurzen und knapp gegliederten Kapiteln beschreibt das Buch die beiden wichtigsten Gruppen der Mykosen, die Hautpilzerkrankungen und die Vaginalmykosen. Die detaillierte Beschreibung der einzelnen Krankheitsbilder in diesen beiden Kapiteln bietet eine hilfreiche Unterstützung der oftmals sehr schwierigen, aber notwendigerweise gezielten und differenzierten Diagnostik. Der Buchteil über Hautmykosen berücksichtigt dabei in erster Linie Pilzerkrankungen der täglichen Praxis. Erfreulicherweise gibt es therapeutische Innovationen in Form neuer Antimykotika und weiterentwickelter Behandlungskonzepte. In den beiden Hauptkapiteln des Buches beschäftigen sich dann getrennte Abschnitte mit den systemisch wirksamen Antimykotika und den Lokalantimykotika. Erprobte Therapiekonzepte helfen dem Leser ganz konkret bei der oft sehr langwierigen und aufwändigen Behandlung der Mykosen. Neben der Darstellung der anerkannten Indikationen wird die Beschreibung der Antimykotika durch eine kurze Darstellung des Wirkmechanismus und des Wirkungsspektrums sowie durch Hinweise auf Erregerlücken sinnvoll ergänzt. Das gut gestaltete Sachwortverzeichnis ermöglicht dem Leser ein schnelles und sicheres Auffinden der gesuchten Begriffe. Besonders hervorzuheben ist die sorgfältige Auswahl qualitativ hochwertiger und einprägsamer Bilder, die das Erkennen typischer Mykosen hilfreich unterstützen sollten. Insgesamt kann das Buch als sehr gelungen bezeichnet werden. Es kann nicht nur jedem Dermatologen empfohlen werden, sondern sollte auch in keiner Praxis eines Pädiaters, Internisten, Gynäkologen oder Hausarztes fehlen. Das Buch kann sicherlich dazu beitragen, den „mykologischen“ Blick zu schärfen, um sich bewusster und wachsamer als bisher mit den mannigfaltigen Erscheinungsformen mykologischer Erkrankungen auseinandersetzen zu können.

Uta-Christina Hipler, Jena 